\title{
Big Data-Infused Service Encounters: Augmenting Emotional Labor and Improving Organizational Outcomes: An Abstract
}

\author{
Jing Chen and Edward Ramirez
}

\begin{abstract}
Combining emotional regulation and role/script theories, this conceptual paper proposes a model that explores how firms can harness insights from big data to augment emotional labor, optimize the service encounter, and thus provide positive outcomes for the firm. Specifically, emotional regulation theory suggests that surface acting decreases and deep acting increases the customer's perceived customer orientation (PCO), resulting in increased positive word of mouth (WOM). Role/script theory proposes that big data analytics provide firms with information that can be used to shield FLEs from emotional dissonance and depletion, moderating the relationships between the strategies used by employees to regulate their emotions and PCO. The paper furthermore argues that the benefits streaming from big data analytics are largely determined by firms' data verification ability. Therefore, managers are urged to effectively leverage big data techniques in managing emotional labor in the service dyad while strengthening their data verification capabilities. Empirical research to quantify the effects of firms' verification ability on successful deployment of big data analytics in the service encounter is encouraged.
\end{abstract}

J. Chen $(\varangle) \cdot$ E. Ramirez

University of Texas at El Paso, El Paso, TX, USA

e-mail: jchen2@miners.utep.edu; eramirez29@utep.edu 